\title{
Characterizing low-coordinated atoms at the periphery of MgO-supported Au islands using scanning tunneling microscopy and electronic structure calculations
}

\author{
Xiao Lin, ${ }^{1}$ Niklas Nilius, ${ }^{1, *}$ Martin Sterrer, ${ }^{1}$ Pekka Koskinen, ${ }^{2}$ Hannu Häkkinen,,${ }^{2,3}$ and Hans-Joachim Freund ${ }^{1}$ \\ ${ }^{1}$ Fritz-Haber Institut der MPG, Faradayweg 4-6, D14195 Berlin, Germany \\ ${ }^{2}$ Department of Physics, Nanoscience Center, University of Jyväskylä, P.O. Box 35 (YFL), FI-40014 Jyväskylä, Finland \\ ${ }^{3}$ Department of Chemistry, Nanoscience Center, University of Jyväskylä, P.O. Box 35 (YFL), FI-40014 Jyväskylä, Finland
}

(Received 16 February 2010; revised manuscript received 12 March 2010; published 15 April 2010)

\begin{abstract}
The perimeter of oxide-supported metal particles is suggested to be of pivotal importance for various catalytic processes. To elucidate the underlying effects, the electronic properties of edge and corner atoms of planar $\mathrm{Au}$ clusters on $\mathrm{MgO} / \mathrm{Ag}(001)$ thin films have been analyzed with scanning tunneling microscopy and electronic structure calculations. The low-coordinated perimeter atoms are characterized by a high density of $s$-derived states at the Fermi level. Those states accommodate transfer electrons from the $\mathrm{MgO} / \mathrm{Ag}$ substrate, which render the perimeter atoms negatively charged. In contrast, the inner atoms of the island are not affected by the charge transfer and remain neutral. This combination of charge accumulation and high state-density explains the specific relevance of the cluster perimeter in adsorption and reaction processes.
\end{abstract}

DOI: $10.1103 /$ PhysRevB.81.153406

PACS number(s): 73.21.La, 68.37.Ef, 68.47.Gh, 71.15.Mb

\section{INTRODUCTION}

Metal aggregates on oxide supports play a decisive role in heterogeneous catalysis. Their outstanding chemical performance involves several aspects. ${ }^{1}$ Metal clusters expose a large number of low-coordinated and hence chemically unsaturated edge and corner atoms that are not available in the respective bulk material. Their electronic structure is determined by quantization effects due to the confined cluster volume. The adclusters also carry extra electrons that originate from a charge transfer either from electron traps in a bulk oxide or from the metal support below a thin oxide film. ${ }^{2,3}$ It has been suggested that these excess charges are in parts responsible for the high catalytic activity of supported metal deposits. ${ }^{4}$

The perimeter atoms of a nanoparticle play a particularly important role for its chemical activity, as they form the boundary between the metal, the oxide support, and the gas phase. ${ }^{5}$ Not surprisingly, various adsorption and reaction phenomena have been identified by theory to occur at the metal-oxide interface. For instance, the periphery atoms of $\mathrm{MgO}$-supported $\mathrm{Au}$ clusters are able to bind $\mathrm{CO}$ with almost $1.0 \mathrm{eV}$, although no adsorption takes place on the cluster top facet. ${ }^{6}$ Similarly, the adsorption and dissociation of $\mathrm{O}_{2}$ is restricted to the low-coordinated edge sites of Au deposits on $\mathrm{TiO}_{2}$ and $\mathrm{MgO} .^{5,7}$ Also the $\mathrm{CO}$ oxidation on a supported catalyst is expected to occur at the metal-oxide interface because the oxide is able to activate the $\mathrm{O}-\mathrm{O}$ bond via a charge transfer while the metal stabilizes the CO. ${ }^{2,5}$ The importance of the cluster perimeter in surface chemistry results from a combination of effects. The boundary atoms are intrinsically of low coordination and therefore subject to specific interaction schemes with their neighbors and the support. They also exhibit a high tendency to accumulate excess charges that have been transferred from the support. ${ }^{8,9}$ Finally, the cluster perimeter is easily accessible for mobile reactants that either diffuse on the metal aggregate or migrate on the oxide surface. This proximity of metal and oxide binding sites renders the periphery atoms ideal candidates to sustain chemical reactions.
The relevance of the cluster perimeter for surface chemistry has been concluded also from experimental evidences, for instance, from the dependence of a certain reaction yield on the length of the cluster-oxide perimeter. ${ }^{10,11}$ However, the distinct coordinative and electronic character of the boundary sites was not directly investigated so far. In particular, it could not be shown to what extent the state density and charge along the perimeter deviate from the cluster interior. The experimental challenge in exploring periphery atoms lies in their small spectral weight when using nonlocal methods. This problem can be circumvented with spatially resolving techniques, such as scanning tunneling microscopy (STM). The STM has proven to be successful in visualizing low-coordinated atoms in nanosized systems, e.g., in $\mathrm{MoS}_{2}$ clusters on $\mathrm{Au}(111)$ (Ref. 12) and $\mathrm{K}$ islands on graphite. ${ }^{13}$ Even so, atomic scale investigations on catalytically relevant metal-oxide systems are still scarce.

In this work, we combine STM and a density-functional tight-binding approach (DFTB) to elucidate the electronic structure and charge state of the periphery atoms of planar $\mathrm{Au}$ islands grown on $\mathrm{MgO} / \mathrm{Ag}(001)$ thin films. Our results provide clear evidence for the special character of the lowcoordinated atom sites, rationalizing their relevance for catalytic processes. ${ }^{4,5,14}$

\section{EXPERIMENT}

The experiments are performed in an ultrahigh vacuum STM operated at $5 \mathrm{~K}^{15}$ A $2-3 \mathrm{ML}$ thick $\mathrm{MgO}$ film is prepared by reactive $\mathrm{Mg}$ deposition onto a sputtered/annealed $\mathrm{Ag}(001)$ surface in $1 \times 10^{-6} \mathrm{mbar}_{2}$ at $570 \mathrm{~K}$. The film completely covers the surface and forms rectangular terraces of several hundred nanometer size. ${ }^{16}$ Gold is evaporated from a high-purity wire wrapped around a tungsten filament onto the cryogenic sample. Subsequent annealing to $150 \mathrm{~K}$ induces the development of planar, beltlike Au islands of $\sim 2 \mathrm{~nm}$ width and up to $50 \mathrm{~nm}$ length [Fig. 1(a)]. ${ }^{17}$ The topographic island height of $0.12 \mathrm{~nm}$ is compatible with a single Au layer bound to the oxide surface. The unusual wet- 


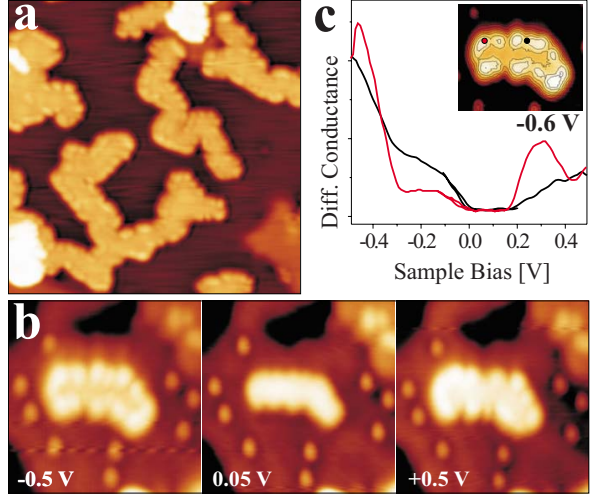

FIG. 1. (Color online) (a) STM images of planar Au islands on $2 \mathrm{ML} \mathrm{MgO} / \operatorname{Ag}(001)\left(U_{\mathrm{S}}=0.2 \mathrm{~V}, 25 \times 25 \mathrm{~nm}^{2}\right)$. (b) Au island and single adatoms imaged at different bias voltages $\left(10 \times 10 \mathrm{~nm}^{2}\right)$. (c) Conductance spectra taken on a kink and a step position of the island shown in the inset. The bias set point was set to $+0.5 \mathrm{~V}$.

ting growth of $\mathrm{Au}$ is triggered by a charge transfer from the $\mathrm{Ag} / \mathrm{MgO}$ interface into the adislands, as described in detail before. ${ }^{3,18}$ The $\mathrm{Au}$ anions strongly bind to the $\mathrm{MgO}$ film via electrostatic and polaronic interactions, which favors the two-dimensional growth of gold. ${ }^{17}$ The electron transfer has been quantified with density functional theory (DFT) to be $0.2|e|$ per $\mathrm{Au}$ atom of a close-packed gold layer on $2 \mathrm{ML}$ $\mathrm{MgO} / \mathrm{Ag}(001){ }^{19}$

\section{PERIMETER ATOMS OF THE GOLD ISLANDS}

STM images taken near the Fermi level $\left(E_{\mathrm{F}}\right)$ reveal no internal structure of the Au islands, indicating their compact morphology [Fig. 1(b)]. However, a sequence of protrusions and depressions becomes visible along the island perimeter. The edge features are most likely of electronic and not of topographic origin, as they emerge only at particular bias voltages. This assumption is verified by differential conductance $(d I / d V)$ maps of the island, which provide an energyresolved measure of the local density of states (LDOS) (Fig. 2). In agreement with the topographic data, pronounced $d I / d V$ contrast develops exclusively at the island edges, while the central region remains dark in the bias window
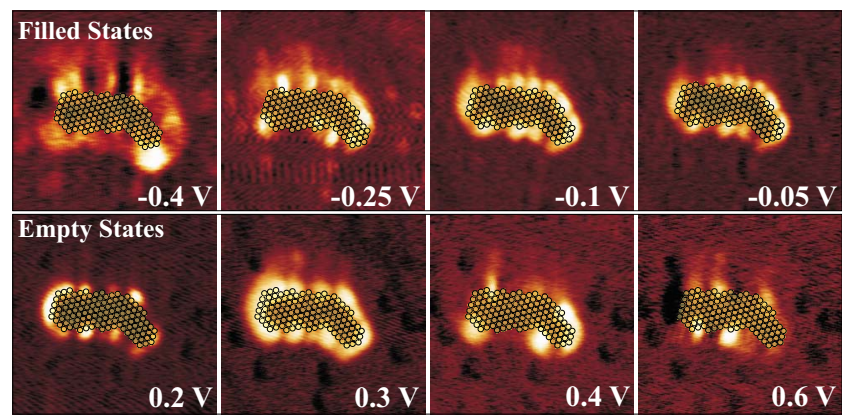

FIG. 2. (Color online). Filled and empty state $d I / d V$ maps of the Au island shown in Fig. 1(b). High $d I / d V$ intensity is only observed along the island edges while the center remains dark at all bias voltages. An atomic model of the island is overlaid. between $\pm 1.0 \mathrm{~V}$. The $d I / d V$ maxima are not pinned to certain perimeter sites but appear on different locations for different bias values. Conductance spectra taken on the island edges show that the $d I / d V$ intensity is confined to two bias regions, ranging from $-0.5 \mathrm{~V}$ to $E_{\mathrm{F}}$ and from +0.2 to $+0.5 \mathrm{~V}$ for the occupied and unoccupied states, respectively [Fig. 1(c)]. The zero-conductance zone separating both regions marks a gap in the electronic states, which disappears in the island center. ${ }^{19}$ It should be noted that the edge features in topographic and $d I / d V$ images occur only above a critical island size of roughly $2 \times 2 \mathrm{~nm}^{2}$ (50 atoms). Smaller islands develop a distinct lobe pattern that is not confined to the edges but extends over the whole island. This lobe structure reflects $\mathrm{Au} 6 s$-like quantum-well states (QWS) that develop at certain energies in the spatially confined system. ${ }^{19}$ For sufficiently symmetric clusters, these QWS adopt flowerlike shapes that resemble the eigenstates of a free-electron gas confined in a parabolic potential. The discrete nature of the QWS fades away with increasing cluster size, as several orbitals with different symmetry overlap in a small energy window. Consequently, the characteristic lobe pattern vanishes and the island interior becomes featureless, as observed in Fig. 1.

The unusual appearance of the island perimeter in the STM is tentatively assigned to a localization of electronic states in low-coordinated edge and corner atoms. To substantiate this interpretation, state-density calculations are performed on the basis of a structure model for the island in Fig. 1(b). Such model cannot be generated by ab initio methods due to the mere cluster size and has therefore been devised empirically by superimposing an $\mathrm{Au}(111)$ sheet to an STM image taken at low bias. The (111) configuration is chosen because of the low free energy of this surface and the lattice orientation is adapted to the direction of distinct island edges observed in the experiment. To account for the limited tip resolution, a $1 \AA$ wide rim is subtracted from the experimental image to obtain a realistic island size. The $\mathrm{MgO}$ lattice is taken directly from atomically resolved STM images. The construction principles applied here were developed and verified on the DFT level for supported $\mathrm{Au}_{8}-\mathrm{Au}_{18}$ clusters. ${ }^{19}$ Figure 3(a) displays the final model for the Au island under investigation. It contains 128 atoms and reproduces not only the overall island shape but also the series of bulges along its upper and lower edges [Fig. 1(b)]. Those maxima and minima are caused by kinks and inverse corners that arise from a small misalignment of the island edges with respect to the (111) lattice. A first validation of the model comes from a comparison with the conductance maps shown in Fig. 2. Although the model is exclusively based on structural information derived from STM images taken in the gap region $(+0.05 \mathrm{~V})$, it perfectly accounts for the $d I / d V$ contrast in filled-state images. The majority of the corners and kinks in the model turn bright at $-0.1 \mathrm{~V}$, while others exhibit $d I / d V$ maxima at slightly lower bias (e.g., the two upper left corners). Those low-coordinated sites also appear bright in empty-state images, although the $d I / d V$ intensity at positive bias is less confined and includes straight sections of the boundary. For example, the edges on the left and right side of the island display a high $d I / d V$ signal at $+0.2 \mathrm{~V}$ and $+0.4 \mathrm{~V}$, respectively. 

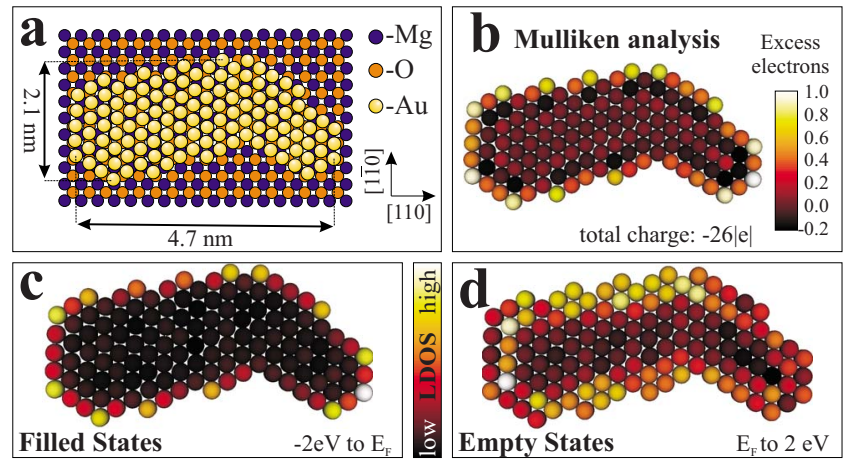

FIG. 3. (Color online) (a) Structure model and (b) charge distribution for the $\mathrm{Au}$ island shown in Fig. 1(b). Mulliken populations from (c) the filled and (d) the empty states within $2 \mathrm{eV}$ below and above the Fermi level, respectively. The model is based on atomically resolved $\mathrm{MgO}$ images and structural features observed for the island.

To capture the underlying physics, the electronic structure of the model island is analyzed with a DFTB approach. ${ }^{20}$ This method is applicable here since it satisfactory describes the metallic bonding and charge distribution in gold. ${ }^{21}$ The comparably weak influence of the $\mathrm{MgO} / \mathrm{Ag}$ substrate is included via the transfer of 0.2 electrons into each interfacial $\mathrm{Au}$ atom, a number that has been derived from $a b$ initio calculations before. ${ }^{3,19}$ The spatial distribution of those transfer electrons within the island is shown in Fig. 3(b). The excess charges localize exclusively at the island perimeter, whereby kink and corner atoms hold almost one extra electron and atoms along straight steps take up half an electron. This charge accumulation at the low-coordinated perimeter sites is compatible with classical electrostatics because it represents the most efficient way to minimize the internal Coulomb repulsion in the island. From a quantum-mechanical viewpoint, the emergence of low-energy states at the perimeter reflects the influence of the repulsive electron-interaction term, which pushes the Au affinity levels out of the system in order to lower the total energy. It should be emphasized that charge transfer into supported Au clusters does not just imply the filling of preexisting states but leads to a substantial reorganization of the LDOS in the system. Even the island shape is affected. As the charge concentration at the perimeter increases linearly with the total number of atoms, it will reach a critical value during island growth above which electron transfer into the $\mathrm{Au}$ is blocked by the Coulomb repulsion. This critical charge amounts to one extra electron per perimeter atom and corresponds to a completely filled $\mathrm{Au} 6 \mathrm{~s}$ state. The $\mathrm{Au} / \mathrm{MgO}$ system avoids this situation by forming elongated, beltlike Au structures with a maximum width of $\sim 2 \mathrm{~nm}$ but up to $50 \mathrm{~nm}$ length [Fig. 1(a)]. By this means, the ratio of inner to perimeter atoms stays constant and charge transfer from the support remains possible.

As the STM is unable to probe the charge state of Au islands directly, the LDOS as an observable quantity has been calculated in addition [Fig. 3(c)]. As expected, the periphery atoms express a high density of filled states with mainly Au $6 s$ character. In correspondence to the experimental $d I / d V$ maps, those states emerge just below $E_{\mathrm{F}}$ and are

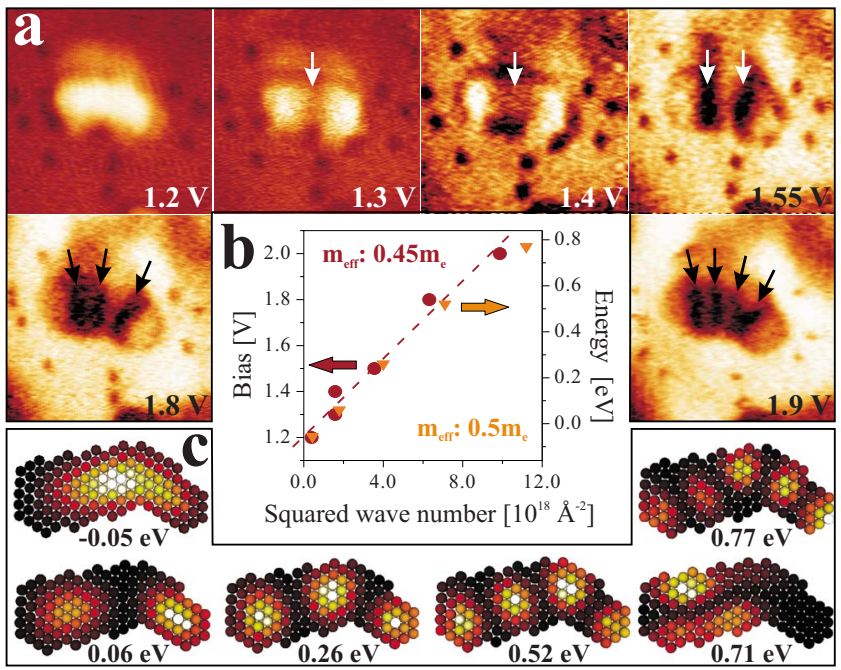

FIG. 4. (Color online) (a) $d I / d V$ maps of the Au island shown in Fig. 1(b) taken at positive bias $\left(10 \times 10 \mathrm{~nm}^{2}\right)$. The $d I / d V$ signal shows an increasing number of vertical nodes at higher bias (see arrows). (b) Dispersion relation derived from the experimental and the calculated density maps. In both cases, a parabolic $E-k$ dependence with an effective electron mass around $0.5 m_{\mathrm{e}}$ is revealed. (c) Calculated state-density plots depicting similar quantization patterns as observed in the $d I / d V$ maps. The computed energies cannot be compared directly to the experimental ones.

therefore well suited to accept the transfer electrons from the $\mathrm{MgO} / \mathrm{Ag}$ support. Also the state density above $E_{\mathrm{F}}$ is enhanced at the periphery, although the empty states protrude more into the island center and extend over several boundary atoms [Fig. 3(d)]. The latter phenomenon might be explained by the development of one-dimensional QWS along the straight boundary sections. The energy of such states depends on the number of involved atoms and the coupling to adjacent steps. High $d I / d V$ intensity is therefore observed at rather different voltages for different step regions (Fig. 2). In contrast, the zero-dimensional kink and corner sites turn bright only in low-bias $d I / d V$ maps, manifesting a localization of states directly at the Fermi level.

\section{QUANTIZATION EFFECTS IN THE INTERIOR OF GOLD ISLANDS}

Quantized electronic states in the island center are observed only at higher positive bias, as demonstrated in the $d I / d V$ series shown in Fig. 4(a). Those QWS become accessible above $+1.2 \mathrm{~V}$ and are assigned to empty $\mathrm{Au} 6 p_{z}$-like states. Whereas at the threshold bias, the $d I / d V$ intensity is evenly distributed within the island; a number of dark lines develops with increasing bias voltage. A first vertical node in the state density emerges at 1.3-1.4 V, while a second, third, and fourth one becomes visible at $1.55 \mathrm{~V}, 1.8 \mathrm{~V}$, and $1.9 \mathrm{~V}$, respectively. The limited stability of the island above $2.0 \mathrm{~V}$ prevents the observation of higher vertical and even horizontal nodes. However, knowledge of the energy position $E_{n}$ and node number $n$ for the lowest five intensity patterns is sufficient to analyze the dispersion relation of the underlying 
electronic states. A plot of the squared wave number $k^{2}$ $=\left[\frac{\pi n}{L}\right]^{2}$, with $L$ the island length, as a function of $E_{n}$ yields a straight line, which is indicative for the parabolic dispersion of a quasifree electron gas [Fig. 4(b)]. Its slope is proportional to the effective electron mass in the potential and has been determined to $0.45 m_{\mathrm{e}}$. DFTB calculations verify the Au $6 p_{z}$ character of the observed QWS and reproduce their characteristic node structure [Fig. 4(c)]. Although absolute QWS energies cannot be acquired due to the ill-defined Fermi level of the charged cluster, the parabolic dispersion relation and the derived effective electron mass of $0.5 m_{\mathrm{e}}$ are in perfect agreement with the STM data. The observed QWS reflect a quantization effect along the long side of the island. As the island width is more than two times smaller, QWS with a horizontal node structure are higher in energy by a factor of (length/width $)^{2} \sim 5$. In the calculations, the first state with a horizontal node appears close to the fifth vertically quantized state, however this state could not be reached within the experimentally accessible bias range.

\section{SUMMARY}

Low-coordinated kink and corner atoms at the perimeter of $\mathrm{Au}$ islands on $\mathrm{MgO} / \mathrm{Ag}(001)$ films have been identified on the basis of their enhanced state density around $E_{\mathrm{F}}$. According to DFTB calculations, those perimeter sites carry the majority of excess electrons that have been transferred from the $\mathrm{MgO} / \mathrm{Ag}$ interface into the adislands. Both effects the localization of electronic states and the accumulation of extra charges render the perimeter atoms the ideal sites for adsorption and reaction events on the $\mathrm{Au} / \mathrm{MgO}$ systems. Our results therefore substantiate the specific role of the boundary between a metal deposit and the oxide support for catalytic processes.

\section{ACKNOWLEDGMENTS}

P.K. and H.H. thank M. Manninen for discussions. This work is supported by the A.v. Humboldt foundation (X.L.), the Academy of Finland, the COST D41 network and the DFG through the "Cluster of Excellence UNICAT."

\footnotetext{
*Corresponding author; nilius@fhi-berlin.mpg.de

${ }^{1}$ Handbook of Heterogeneous Catalysis, edited by G. Ertl, H. Knözinger, F. Schueth, and J. Weitkamp (Wiley-VCH, Weinheim, 2008).

${ }^{2}$ B. Yoon, H. Häkkinen, U. Landman, A. S. Wörz, J. Antonietti, S. Abbet, J. Judai, and U. Heiz, Science 307, 403 (2005).

${ }^{3}$ G. Pacchioni, L. Giordano, and M. Baistrocchi, Phys. Rev. Lett. 94, 226104 (2005); M. Sterrer, T. Risse, U. M. Pozzoni, L. Giordano, M. Heyde, H.-P. Rust, G. Pacchioni, and H.-J. Freund, ibid. 98, 096107 (2007).

${ }^{4}$ T. Risse, S. Shaikhutdinov, N. Nilius, M. Sterrer, and H.-J. Freund, Acc. Chem. Res. 41, 949 (2008).

${ }^{5}$ L. M. Molina and B. Hammer, Appl. Catal., A 291, 21 (2005).

${ }^{6}$ S. Sicolo, L. Giordano, and G. Pacchioni, J. Phys. Chem. C 113, 10256 (2009).

${ }^{7}$ P. Frondelius, H. Häkkinen, and K. Honkala, Phys. Chem. Chem. Phys. 12, 1483 (2010)

${ }^{8}$ P. Frondelius, H. Häkkinen, and K. Honkala, Phys. Rev. B 76, 073406 (2007); New J. Phys. 9, 339 (2007).

${ }^{9}$ V. Simic-Milosevic, M. Heyde, X. Lin, T. König, H.-P. Rust, M. Sterrer, T. Risse, N. Nilius, H.-J. Freund, L. Giordano, and G. Pacchioni, Phys. Rev. B 78, 235429 (2008).

${ }^{10}$ J. A. Rodriguez, S. Ma, P. Liu, J. Hrbek, J. Evans, and M. Perez, Science 318, 1757 (2007); P. Liu and J. A. Rodriguez, J. Chem. Phys. 126, 164705 (2007).

${ }^{11}$ J. Kibsgaard, K. Morgenstern, E. Lægsgaard, J. V. Lauritsen, and
}

F. Besenbacher, Phys. Rev. Lett. 100, 116104 (2008).

${ }^{12}$ M. V. Bollinger, J. V. Lauritsen, K. W. Jacobsen, J. K. Nørskov, S. Helveg, and F. Besenbacher, Phys. Rev. Lett. 87, 196803 (2001).

${ }^{13}$ F. Yin, J. Akola, P. Koskinen, M. Manninen, and R. E. Palmer, Phys. Rev. Lett. 102, 106102 (2009).

${ }^{14}$ M. Haruta, Catal. Today 36, 153 (1997); Chem. Rec. 3, 75 (2003).

${ }^{15}$ H.-P. Rust, J. Buisset, E. K. Schweizer, and L. Cramer, Rev. Sci. Instrum. 68, 129 (1997).

${ }^{16}$ S. Schintke, S. Messerli, M. Pivetta, F. Patthey, L. Libioulle, M. Stengel, A. De Vita, and W. D. Schneider, Phys. Rev. Lett. 87, 276801 (2001).

${ }^{17}$ M. Sterrer, T. Risse, M. Heyde, H.-P. Rust, and H.-J. Freund, Phys. Rev. Lett. 98, 206103 (2007).

${ }^{18}$ D. Ricci, A. Bongiorno, G. Pacchioni, and U. Landman, Phys. Rev. Lett. 97, 036106 (2006).

${ }^{19}$ X. Lin, N. Nilius, H.-J. Freund, M. Walter, P. Frondelius, K. Honkala, and H. Häkkinen, Phys. Rev. Lett. 102, 206801 (2009).

${ }^{20} \mathrm{P}$. Koskinen and V. Mäkinen, Comput. Mater. Sci. 47, 237 (2009).

${ }^{21}$ We used the open-source software HOTBIT (https://trac.cc.jyu.fi/ hotbit). The Au-Au parameterization is described in P. Koskinen, H. Häkkinen, G. Seifert, S. Sannan, T. Frauenheim, and M. Moseler, New J. Phys. 8, 9 (2006). 